University of New Hampshire

University of New Hampshire Scholars' Repository

Molecular, Cellular and Biomedical Sciences

Scholarship

Molecular, Cellular and Biomedical Sciences

$1-28-2010$

\title{
Diarylamidines: high potency inhibitors of acid-sensing ion channels
}

\author{
Xuanmao Chen \\ University of New Hampshire, Durham, Xuanmao.Chen@unh.edu \\ Liyan Qiu \\ University of Toronto \\ Minghua Li \\ University of Toronto \\ Stefan Grunder \\ Aachen University \\ Beverley A. Orser \\ University of Toronto
}

See next page for additional authors

Follow this and additional works at: https://scholars.unh.edu/mcbs_facpub

Comments

This is an Accepted Manuscript of an article published by Elsevier in Neuropharmacology in 2010, available online:

https://dx.doi.org/10.1016/j.neuropharm.2010.01.011.

\section{Recommended Citation}

Chen X, Qiu L, Li M, Durrnagel S, Orser BA, Xiong ZG, MacDonald JF. Diarylamidines: high potency inhibitors of acid-sensing ion channels. Neuropharmacology. 2010;58(7):1045-53. doi: 10.1016/ j.neuropharm.2010.01.011. PubMed PMID: 20114056; PubMed Central PMCID: PMC3846926.

This Article is brought to you for free and open access by the Molecular, Cellular and Biomedical Sciences at University of New Hampshire Scholars' Repository. It has been accepted for inclusion in Molecular, Cellular and Biomedical Sciences Scholarship by an authorized administrator of University of New Hampshire Scholars' Repository. For more information, please contact Scholarly.Communication@unh.edu. 
Authors

Xuanmao Chen, Liyan Qiu, Minghua Li, Stefan Grunder, Beverley A. Orser, Zhi-Gang Xiong, and John F. MacDonald 
Published in final edited form as:

Neuropharmacology. 2010 June ; 58(7): . doi:10.1016/j.neuropharm.2010.01.011.

\title{
Diarylamidines: High potency inhibitors of acid-sensing ion channels
}

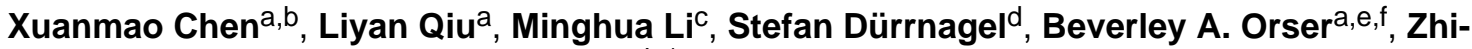
Gang Xiong $^{\mathrm{c}}$, and John F. MacDonald ${ }^{\mathrm{a}, \mathrm{b},{ }^{*}}$

aDepartment of Physiology, University of Toronto, Canada

${ }^{b}$ Robarts Research Institute, University of Western Ontario, Canada

'Robert S. Dow Neurobiology Laboratories, Legacy Research, Portland, USA

dDepartment of Physiology, RWTH Aachen University, Germany

eSunnybrook Health Sciences Centre, University of Toronto, Ontario, Canada

fDepartment of Aesthesia, University of Toronto, Ontario, Canada

\begin{abstract}
Acid-sensing ion channels (ASICs) are proton-gated cation channels that are predominantly expressed in the nervous system. ASICs are involved in a number of neurological diseases such as pain, ischemic stroke and multiple sclerosis but limited tools are available to target these channels and provide probes for their physiological functions. Here we report that the anti-protozoal diarylamidines, 4',6-diamidino-2-phenylindole (DAPI), diminazene, hydroxystilbamidine (HSB) and pentamidine potently inhibit ASIC currents in primary cultured hippocampal neurons with apparent affinities of $2.8 \mu \mathrm{M}, 0.3 \mu \mathrm{M}, 1.5 \mu \mathrm{M}$ and $38 \mu \mathrm{M}$, respectively. These four compounds $(100 \mu \mathrm{M})$ failed to block ENaC channels expressed in oocytes. Sub-maximal concentrations of diminazene also strongly accelerated desensitization of ASIC currents in hippocampal neurons. Diminazene blocked ASIC1a, $-1 \mathrm{~b}-2 \mathrm{a}$, and -3 currents expressed in $\mathrm{CHO}$ cells with a rank order of potency $1 \mathrm{~b}>3>2 \mathrm{a} \geq 1 \mathrm{a}$. Patchdock computational analysis suggested a binding site of diarylamidines on ASICs. This study indicates diarylamidines constitute a novel class of nonamiloride ASIC blockers and suggests that diarylamidines may be developed as therapeutic agents in treatment of ASIC-involved diseases.
\end{abstract}

\section{Keywords}

ASIC; Diarylamidines; Channel blockers; Pain

\section{Introduction}

ASICs represent a 6-member (ASIC1a, -1b, -2a, -2b, -3 and -4) subgroup of the degenerin/ epithelial sodium channel (DEG/ENaC) family of cation channels (Grunder et al., 2000; Waldmann et al., 1997; Wemmie et al., 2006) and they are widely expressed in sensory neurons of the dorsal root ganglion (DRG) and in the neurons of the brain (Lingueglia, 2007; Xiong et al., 2008). The crystal structures of chicken ASIC1 have been resolved

Copyright $@ 2010$ Published by Elsevier Ltd. All rights reserved.

*Corresponding author at: Robarts Research Institute, University of Western Ontario, 100 Perth Drive, London, ON, Canada N6A 5K8. Tel.: +1 519 6635777; fax: +1 519 9315721. jfmacdonald@ robarts.ca (J.F. MacDonald). 
(Gonzales et al., 2009; Jasti et al., 2007). These channels are implicated in a wide spectrum of neuronal functions including synaptic plasticity, mechanosensation and modulation of retinal function (Krishtal, 2003; Wemmie et al., 2006). However, the normal physiological functions of ASIC remain not fully understood, partly due to the lack of good pharmacological tools for their study.

In contrast, ASICs have been extensively studied for their pathophysiological roles. ASICs contribute to several disease states. For example, activation of ASIC1a expressed in interneurons is thought to facilitate termination of epileptic seizure when $\mathrm{pH}$ falls (Ziemann et al., 2008). ASIC1a is also associated with axonal degeneration in the experimental autoimmune encephalomyelitis (EAE) model (Friese et al., 2007) and contributes to focal ischemic brain injury (Xiong et al., 2004). In peripheral neurons ASICs are widely believed to mediate acid-induced pain or to act as sensors of inflammatory pain (Deval et al., 2008). ASIC inhibitors attenuate peripheral acid-induced hyperalgesia in rats (Dube et al., 2005) as well as pain in human subjects (Ugawa et al., 2002).

There are few ASIC inhibitors available to probe their function in vivo or to intervene in ASIC-associated diseases. Two poly-peptide toxins were identified as modulators of ASIC activity (Chen et al., 2005; Diochot et al., 2004; Escoubas et al., 2000) and several small molecules, in addition to the diuretic amiloride and derivatives (Kuduk et al., 2009a,b), have been reported to inhibit ASIC currents. Some non-steroidal anti-inflammatory drugs inhibit ASICs (Voilley et al., 2001). A-317567 and nafamostat mesilate also block native ASIC currents in dorsal root ganglion (DRG) neurons and oocyte-expressing ASICs (Dube et al., 2005; Ugawa et al., 2007) but do so with relatively low potency.

Here we report that a class of anti-protozoan diarylamidines including diminazene, DAPI, $\mathrm{HSB}$, and pentamidine inhibit ASIC currents in cultured hippocampal neurons with relatively high affinity. They also attenuate the excitation of these neurons in response to an acid challenge. Moreover, diminazene dramatically enhances the rate of desensitization of ASIC currents. Based on structural-functional analysis of diarylamidines, their analogs, and ASICs, we mapped out a common molecular template for designing potent blockers for ASICs. This novel class of ASIC blockers might be useful for ASIC functional study and be of therapeutic significance for intervention in ASIC-related disorders such as chronic pain.

\section{Methods}

\subsection{Cell culture}

All animal experiments were carried out according to guidelines approved by the University of Toronto Animal Care Committee. Primary cultures of mouse hippocampal and cortical neurons were prepared as previously described (Wei et al., 2007). Briefly, cultures of hippocampal neurons were prepared from Swiss mice. Pregnant mice were sacrificed by cervical dislocation under anesthesia (isoflurane) and fetuses were rapidly removed at embryonic day E17-18. Hippocampal tissues were dissected and placed in cold Hanks' solution prior to dissociation. Hippocampal neurons were dissociated by mechanical trituration and plated on $35 \mathrm{~mm}$ culture dishes at an estimated density of $<1 \times 10^{6} \mathrm{cells} / \mathrm{cm}^{2}$. Cell cultures were incubated in a minimal essential media supplemented with $10 \%$ fetal bovine serum, $10 \%$ inactivated horse serum and insulin $(8 \mu \mathrm{g} / \mathrm{ml})$ at $37^{\circ} \mathrm{C}$ in $5 \%$ carbon dioxide for the first week (all chemicals from Invitrogen, Carlsbad, CA, USA). Fibroblast/ glial cell proliferation was terminated by the addition of floxuridine at day 7 in vitro. 


\subsection{Electrophysiological recording on cultured hippocampal neurons}

Electrophysiological recordings were performed on primarily cultured mouse hippocampal neurons 14-22 days after plating. The extracellular solution was composed of (mM) 140 $\mathrm{NaCl}, 2 \mathrm{CaCl}_{2}, 1 \mathrm{MgCl}_{2} 25 \mathrm{~N}$-2-Hydroxyethylpiperazine- $\mathrm{N}^{\prime}$-thanesulfonic acid (HEPES), 33 glucose, $5.4 \mathrm{KCl}$ and 0.0002 Tetrodotoxin with $\mathrm{pH}$ of 7.3-7.4 and osmolarity ranging from 320 to $330 \mathrm{mOsm}$. The intracellular solution for voltage clamp recording consisted of (mM) $140 \mathrm{CsF}, 11$ Ethyleneglycol-bis-(a-amino-ethyl ether) N,N'-tetra-acetic acid (EGTA) as intracellular calcium chelating buffer, $10 \mathrm{HEPES}, 2 \mathrm{MgCl}_{2}$, 2 Tetraethyl ammonium chloride (TEA-Cl), $1 \mathrm{CaCl}_{2}$, and $4 \mathrm{~K}_{2} \mathrm{ATP}$ with $\mathrm{pH}$ adjusted by $\mathrm{CsOH}$. For current-clamp recording the pipette solution contained (inmM): $140 \mathrm{KCl}, 5 \mathrm{NaCl}, 10 \mathrm{HEPES}, 5$ EGTA, 2 $\mathrm{MgCl}_{2} 2 \mathrm{~K}_{2} \mathrm{ATP}, \mathrm{pH}$ was adjusted by $\mathrm{KOH}$. Pipette resistance ranges $2-4 \mathrm{M} \Omega$. Recordings were performed at room temperature. The membrane potential was held at $-60 \mathrm{mV}$ throughout the recordings unless otherwise indicated. Access resistance was monitored by applying a voltage step of $-5 \mathrm{mV}$. ASIC mediated currents were elicited by rapid application of pH 5.8 (low pH buffer, 2-N-morpholino-ethanesulfonic acid (MES) replacing HEPES) delivered from a multi-barrel fast perfusion system for $5 \mathrm{~s}$ in every minute. The solution perfusion rate was approximately $1 \mathrm{ml}$ per minute. Whole-cell currents were recorded using an Axopatch-1D amplifier (Molecular Devices, Sunnyvale, CA). Electrophysiological data were filtered at $2 \mathrm{kHz}$ and digitized at $5-10 \mathrm{kHz}$ using a Digidata 1332A or/and simultaneously through MiniDigi 1A, and acquired online with pClamp8.2 (Axon Instruments) or/and Axoscope9.2 (Axon instruments).

\subsection{Transfection of Chinese hamster ovary cells $(\mathrm{CHO})$ and electrophysiological recording}

Transfection of CHO cells was performed as described previously (Chu et al., 2004), Briefly, CHO cells were cultured in F12 medium (American Type Culture Collection, Manassas, VA) supplemented with 10\% fetal bovine serum. At 50\% confluence, cells were cotransfected with DNA for various ASICs and for green fluorescent protein (GFP) in the pcDNA3 vector (Invitrogen, San Diego, CA), using Fugene 6 transfection reagent (Roche Diagnostics, Indianapolis, IN). For each $35 \times 35 \mathrm{~mm}$ culture dish, $1.8 \mu \mathrm{g}$ of cDNA for individual ASIC and $0.2 \mu \mathrm{g}$ ofDNA for GFP were used. All recordings were made $48-72 \mathrm{~h}$ after plating. GFP-positive cells were viewed under a fluorescence microscope for patchclamp recording. For all ASIC subunits, a holding potential of $-60 \mathrm{mV}$ was used. Low $\mathrm{pH}$ values close to $\mathrm{pH} 50$ of individual ASIC subunits (e.g. 6.0 for ASIC1a and 1b, 5.0 for ASIC2a, and 6.5 for ASIC3) were used to activate the ASIC currents.

\subsection{Electrophysiology using two-electrode voltage clamp}

Experimental procedures for whole oocyte electrophysiology have been previously described (Babini et al., 2003; Paukert et al., 2004). Ovaries of adult Xenopus laevis females were surgically removed under anesthesia. The follicular membrane was removed by digestion with collagenase type II (Sigma; $1 \mathrm{mg} \mathrm{ml}^{-1}$ ) for 60-120 min. Capped cRNAs of $\mathrm{ENaC}$ subunits were synthesized by SP6 RNA polymerase from cDNA of ENaC alpha, beta and -gamma subunits using the mMessage mMachine kit (Ambion). $1 \mathrm{ng}$ of cRNA of EnaC was injected into stage V-VI oocytes of $X$. laevis. Oocytes were kept in a low $\mathrm{Na}^{+}$ oocyte ringer-2 medium ( $5 \mathrm{~mm} \mathrm{NaCl}, 77.5 \mathrm{~mm} \mathrm{~N}$-methyl-d-glucamine, $1 \mathrm{~mm} \mathrm{Na} 2 \mathrm{HPO}_{4}, 2.5$ $\mathrm{mm} \mathrm{KCl}, 5 \mathrm{~mm}$ HEPES, $0.5 \mathrm{~g} / \mathrm{l}$ polyvinylpyrrolidone, $1 \mathrm{~mm} \mathrm{MgCl}_{2}$, and $1 \mathrm{~mm} \mathrm{CaCl}_{2}$ ) at 19 ${ }^{\circ} \mathrm{C}$ for 3 days. During recording, oocytes were superfused with ringer solution containing $140 \mathrm{~mm} \mathrm{NaCl}, 2.5 \mathrm{~mm} \mathrm{KCl}, 1.8 \mathrm{~mm} \mathrm{CaCl}_{2}, 1 \mathrm{mM} \mathrm{MgCl}_{2}$ and $10 \mathrm{~mm}$ HEPES (pH 7.4). Amiloride-sensitive current was measured using two-electrode voltage clamp. To determine the effect of diarylamidines on $\mathrm{ENaC}$ activities, oocytes were superfused first with a solution containing $10 \mu \mathrm{m}$ amiloride, which was then replaced with solutions containing 0 $\mu \mathrm{m}$ amiloride. Diarylamidines $(100 \mu \mathrm{M})$ were applied at the absence of amiloride. Whole 
oocyte amiloride-sensitive currents were recorded at $1 \mathrm{kHz}$ with a TurboTec $03 \mathrm{X}$ amplifier (npi electronic). Solutions were applied by means of a fast, automated perfusion system (Screening tool, npi electronic). Holding potential was $-70 \mathrm{mV}$ and all experiments were performed at room temperature $\left(22-26^{\circ} \mathrm{C}\right)$. Data acquisition was managed using the software CellWorks 5.1.1 (npi electronic).

\subsection{Docking diarylamidines onto ASIC1 crystal structures}

The crystal structure of cASIC1 was obtained from Protein Data Band code 3HGC (Gonzales et al., 2009) or from 2QTS (Jasti et al., 2007) as a comparison. The structures of 4 novel blockers (diminazene, DAPI, hydroxystilbamidine, pentamidine) as well as nafamostat were downloaded from PDB bank. Rather than perform homology modeling of rat ASIC based on cASIC 1 crystals in advance, we directly docked these chemicals into cASIC1 crystals (3HGC) to minimize possible distortion or miscalculation derived from homology modeling. We uploaded structures of ASIC1 (structure of a single subunit and complete trimeric channel, respectively) and blockers onto the Patchdock server (http:// bioinfo3d.cs.tau.ac.il/PatchDock/). Docking was performed by the server between cASIC1 and each of the blockers in turn and docking results with various possibility scores were sent back via email. We chose and analyzed only docking results with high scores. Docked structures were visualized and rendered using Pymol (www.pymol.org).

\subsection{Data analysis and chemicals}

Data were analyzed with Clampfit 9.2. (Axon Instruments), Excel 2002 (Microsoft Corporation), Origin 5.0 (Microcal Software) and illustrated using CorelDraw13 (Corel Corporation). Currents were normalized to the amplitude of control responses. Inhibitory concentration-response plots were fitted to the logistic equation: $I=\left(A_{\max }-A_{0}\right) /[1+(X /$ $\left.\left.\mathrm{IC}_{50}\right)^{n}\right]+A_{0}$, where $I$ is the normalized current amplitude, $X$ was the antagonist concentration; $n$ is Hill coefficient; $\mathrm{IC}_{50}$ is the concentration of antagonist that generate $50 \%$ of maximal inhibition. All data points were expressed as mean \pm S.E.M. Paired Student's $t$ test was used to examine the statistical significance of the difference between groups of data. The criterion of significance was set at $p<0.05$.

Structures presented in figures were re-drawn based on the structures from Pubchem (http:// www.ncbi.nlm.nih.gov/sites/entrez). Diminazene, pentamidine, gabexate, DAPI, HSB were purchased from Sigma-Aldrich (Oakville, ON, Canada). Synthalin was from BioMol (Plymouth meeting, PA, USA).

\section{Results}

\subsection{Identification of ASIC blockers}

To identify novel ASIC inhibitors, we performed a small scale screening. As the ectodomain of ASICs contain around 80 charged amino acids (about half of them are conserved) among total 360 amino acids (Paukert et al., 2008), we hypothesized that poly- or bi-charged chemicals are likely to bind to ASIC channels. We first tested suramin, a poly-anion, but suramin $(500 \mu \mathrm{M})$ failed to inhibit ASIC currents in cultured hippocampal neurons. Next we examined bi-charged compounds. We tested if a negatively charged di-anion can modulate ASICs currents. Carbenoxolone, a blocker of pannexin, is such a di-anion. However, at 200 $\mu \mathrm{M}$ it also failed to inhibit ASICs (Fig. 1A). Then we assessed positively charged netropsin and synthalin, linear diguaninines, for their effects on ASIC currents. Synthalin $(200 \mu M)$ weakly inhibited ASIC currents by $29 \pm 3 \%$ ( $n=6$, Fig. 1B) whilst netropsin was without effect. As synthalin is a positively charged molecule, we then tested if diamidines, which share comparable size and similar charge pattern as diguanidines, might have similar actions on ASICs. We first studied imidocarb, a derivative from amicarbalide that is a diamidine 
(Wilson et al., 2005). Imidocarb $(200 \mu \mathrm{M})$ which weakly inhibited $42 \pm 7 \%(n=5)$ ASIC currents (Fig. 1D), has two positive charges that are not freely movable at its ends.

\subsection{Diarylamidines potently and reversibly inhibits ASIC currents}

Next we determined if the anti-protozoal agents, the diary-lamidines, inhibit ASIC currents in cultured hippocampal neurons. Diarylamidines are a large family of biochemically and pharmacologically interesting synthetic compounds which includes DAPI, HSB, pentamidine, diminazene etc.(Baraldi et al., 2004). Indeed, we found that these diarylamidines strongly inhibited ASIC currents. Fig. 2 shows that HSB inhibited ASIC currents in hippocampal neurons with an $\mathrm{IC}_{50}$ of $1.5 \pm 0.6 \mu \mathrm{M}(n=5)$. Pentamidine inhibited ASIC with an $\mathrm{IC}_{50}$ of $38 \pm 11 \mu \mathrm{M}(n=4)$ and DAPI inhibited with an $\mathrm{IC}_{50}$ of $2.8 \pm 0.7 \mu \mathrm{M}$ $(n=4)$. Fig. 3 shows that diminazene potently blocked ASIC channels in cultured hippocampal neurons with a potency of $0.29 \pm 0.11 \mu \mathrm{M}(n=6)$. Diminazene is the most potent small-molecular inhibitor of ASIC known so far. In addition to the reduction of current amplitude, sub-maximal concentrations of diminazene also facilitated the rate of desensitization of ASIC currents. Fig. 3B shows that co-application of diminazene ( $3 \mu \mathrm{M})$ with $\mathrm{pH} 5.8$ solution drastically $(p<0.01)$ reduced the desensitization time constant of ASIC currents from $1.6 \pm 0.3 \mathrm{~s}$ to $0.4 \pm 0.1 \mathrm{~s}(n=6)$. This suggests that diminazene may promote the closure of ASIC channels. In contrast, sub-maximal concentrations of HSB, pentamidine and DAPI did not have marked effects on rates of desensitization of ASIC currents (not shown).

We also compared the effects of diminazene on ASIC desensitization with sub-maximal concentration of amiloride. Amiloride is an open channel blocker of ASICs (Paukert et al., 2004). The $\mathrm{IC}_{50}$ of amiloride on ASIC current is reported to be 10-20 $\mu \mathrm{M}$ (Waldmann et al., 1997; Wu et al., 2004) but up to 200-500 $\mu \mathrm{M}$ amiloride is required to completely block the neuronal ASIC currents (Baron et al., 2002; Wu et al., 2004). Therefore, we used a submaximal $(80 \mu \mathrm{M})$ with co-application $\mathrm{pH} 5.8$ solution to inhibit a large part, but not all of the ASIC current. We observed little effect of amiloride on desensitization of ASIC current. Fig. 3C demonstrates that amiloride only reduced the desensitization time constant of ASIC currents from $0.83 \pm 0.15 \mathrm{~s}$ to $0.72 \pm 0.14 \mathrm{~s}(n=9, p=0.28)$. These results suggest that diminazene inhibits ASIC currents via a distinct mechanism compared to amiloride and implies that diminazene and amiloride represent different classes of ASIC channel inhibitors.

We also found that the facilitation of desensitization of these currents by diminazine was dependent upon its concentration. The greatest change occurred with $1 \mu \mathrm{M}$ diminazine which changed the desensitization time constant from $1.7 \pm 0.2$ to $0.4 \pm 0.1 \mathrm{~s}(n=13, p<$ 0.01 , Fig. 3D). Fig. 3D also suggests that the effect of diminazene increased when the initial rate of desensitization of ASIC current was slower. This suggests that individual subtypes of ASICs might have different sensitivities to diminazene as recombinant ASIC receptors demonstrate substantially different rates of desensization.

As ASIC and ENaC proteins share considerable homology (Kellenberger and Schild, 2002), we heterologously expressed ENaC channels in Xenopus oocytes and used two-electrode voltage clamp techniques to test if diarylamidines block ENaC activities. Fig. 4A shows that none of these four diarylamidines (DAPI, HSB, pentamidine and diminazene; $n=8$ for each) inhibit ENaC current in oocytes at a concentration of $100 \mu \mathrm{M}$. But at this concentration DAPI $(n=5)$, diminazene $(n=5)$ and HSB $(n=5)$ blocked ASIC1a currents in oocytes almost completely. For pentamidine $(n=5)$ a block of $77 \%$ on ASIC1a was observed. These data indicate that diaryamidines act selectively on ASIC channel over $\mathrm{ENaC}$. Low $\mathrm{pH}$ stimuli induce action potentials and membrane depolarization by activating ASICs (Baron et al., 2002). To determine whether diminazene could inhibit this acid- 
induced increase in excitability, we performed current-clamp recordings from cultured hippocampal neurons. Fig. 4B shows that pH 6 markedly depolarized neurons and evoked a train of action potentials. Application of diminazene $(10 \mu \mathrm{M})$ dramatically attenuated the $\mathrm{pH}$ 6-induced depolarization and excitation $(n=7)$ illustrating that this medicinal compound could be used to attenuate acid-induced over-excitation by inhibiting ASIC activities. Diminazene $(10 \mu \mathrm{M})$ itself had little effect on firing frequency (Fig. 4C, $n=4)$, implying that it is unlikely to affect voltage-gated ion currents underlying the generation of action potentials in these neurons.

We next heterologously expressed individual ASIC subtypes in CHO cells and assessed the effect of diminazene on ASIC currents mediated by individual ASIC subunits. We first tested the effect of diminazene on the ASIC1a current. A preliminary concentrationresponse analysis revealed an $\mathrm{IC}_{50}$ of $\sim 3 \mu \mathrm{M}$ for the inhibition of the ASIC1a current by diminazene ( $n=2$, not shown). Therefore, in the subsequent experiments, $3 \mu \mathrm{M}$ diminazene was used to compare its effect on ASIC currents mediated by ASIC1a, $-1 \mathrm{~b},-2 \mathrm{a}$, and -3 subunits. Addition of diminazene $(3 \mu \mathrm{M})$ in both the conditioning solution $(\mathrm{pH} 7.4)$ and the low $\mathrm{pH}$ solutions dramatically reduced the amplitude of ASIC1a, $-1 \mathrm{~b},-2 \mathrm{a}$, and -3 currents (Fig. 5). Following a 2 min perfusion of diminazene, ASIC1 a current was inhibited by $39 \pm$ $3.8 \%$; ASIC 1 b by $92 \pm 0.5 \%$; ASIC2a by $51 \pm 6.1 \%$; and ASIC 3 by $74 \pm 0.6 \%$. The inhibition of the ASIC currents by diminazene was completely reversible (Fig. 5). Therefore, diminazene most potently blocks ASIC1b, an ASIC subunit exclusively expressed in sensory neurons (Chen et al., 1998).

\subsection{Docking diarylamidines onto the crystal structure of ASICs suggests a potential common binding region}

DAPI, HSB and pentamidine and diminazene are linear divalent cations which are determined by their amidine groups at both ends, with variable bulk (i.e. benzene rings) on the outer edge of the molecule. They are semi-symmetric with two diarylamidines connected by molecular bridges of varying length and composition. They could probably bind to the same domain of ASICs due to their similar structural pattern. We therefore used in-silicon "Patchdock" analysis to study where these compounds bind to ASIC by making use of recently solved cASIC1 crystal structure (Gonzales et al., 2009). Patchdock can provide computational analysis and predict the docking of ligands to their receptors, yielding a variety of binding possibilities with scores ranking from high to low. First we analyzed binding possibilities using one subunit of ASIC1 structure. Although there are different binding possibilities for each individual inhibitor, all four diarylamidines docked onto one common region (Fig. 6A). In addition, docking possibilities for these common regions generated the highest scores, suggesting this region might likely be the binding domain. Patchdock analysis implied that these diarylamidines bind to a small groove in the surface of cASIC1 crystal, which is formed by part of the beta-ball domain and the palm domain. The bottom part of the groove is formed mostly by hydrophobic amino acids (Fig. 6B), which thereby create a hydrophobic micro-environment. The dumbbell-shaped diarylamidine blockers fit well into this groove. The hydrophobic groove is consistent with the structural properties of diarylamidines which are mostly hydrophobic, except for their charged diamidine termini, composed of aromatic rings connected with hydrophobic linkage. The top of the groove constitutes a narrow gate (with an opening of $4.9 \AA$ ) controlled by K205 and D184, which probably allow the longitudinal diarylamidines (length varying from 12 to 19 $\AA$ ) to bind into the groove from one angle (Fig. 6B). To avoid over-simplification of the patchdock based on single subunit, we also performed patchdock computations using the full trimeric ASIC1 crystal structures. Consistently these computations also suggested that diarylamidines likely bind to the same region, although the probability scores were not as high as that using single subunit. Fig. $6 \mathrm{C}$ shows that diminazene docks onto the full trimeric 
channel. Docking experiments suggested a potential common binding region of diarylamidines on ASIC. Nevertheless, we could not rule out that diarylamidines might bind to other domains.

\section{Discussion}

In this study we first report that diarylamidines inhibit ASIC currents with high potencies compared to known ASIC inhibitors such as amiloride. Diarylamidines are a large family of synthetic compounds. Currently this chemical family has more than 30 members and its family number is rapidly increasing (Baraldi et al., 2004). Diarylamidines have been widely used for the treatment of protozoan diseases such as trypanosomiasis and leishmaniasis since 1930s (Baraldi et al., 2004; Mishra et al., 2007; Rosenthal, 2007). We tested a few agents and report that four agents potently inhibit ASIC currents and it is conceivable that other diarylamidines would inhibit ASIC activities. Pentamidine is the best known member of the diarylamidines because of its activity against trypanosomatid protozoans and Pneumocystis jirovecii. Hydroxystilbamidine had been used in the treatment of nonprogressive blastomycosis of the skin and other mycoses (Hermans and Keys, 1983). Diminazene is commonly used in veterinary medicine for the treatment of trypanosomiasis in domestic livestock (Baraldi et al., 2004; Kinabo, 1993). DAPI is widely used as a fluorescence marker for chromosomal DNA, a classic minor groove binder at A/T sequence (Baraldi et al., 2004). DAPI demonstrates strong anti-trypanosome activity but its clinical use as an anti-protozoal agent has been limited due to its strong undesirable cytotoxic side effects (Baraldi et al., 2004). Other agents like pentamidine also demonstrate significant cytotoxicity but it remains in use clinically (Rosenthal, 2007). The anti-protozoan mechanism of diarylamidines is not yet clearly known. Proposed anti-protozoan mechanisms include DNA-binding to the minor groove (Wilson et al., 2005), because DAPI, diminazene, pentamidine, and HSB all bind to the minor groove of DNA.

As the ASIC crystal structure has been solved (Gonzales et al., 2009), the "Patchdock" computation allowed us to dock DAPI, diminazene, HSB and pentamidine, respectively to the ASIC1 crystal structure and analyze possible binding domain of these diarylamidines. Our analysis suggests that these four compounds likely bind to one region of ASICs and that region is located at the beta-ball and palm domain. We also experimented with nafamostat using Patchdock analysis and found nafamostat also docks onto the same region as diarylamidines (not shown), supporting the hypothesis that these similar di-cationic compounds share the same binding region on ASICs. Nevertheless, docking analysis has its shortcoming. It only suggests several binding possibilities and it cannot explicitly indicate a binding site. Therefore we cannot rule out the possibility that other binding regions exist. Indeed, some other possible binding sites are suggested by the patchdock, for example, one possible binding site is a cavity, formed by residues S252, E255, L262, S305, I306, N367, V368, T369 and R370. But these sites are less likely than the one we have shown, because not all compounds dock there. To definitely confirm the binding site, however, mutagenesis experiments are required.

Our data show that diminazene influences ASIC desensitization while amiloride does not. In addition, diarylamidines are structurally distinct from amiloride (Table 1). Diarylamidines constitute a novel class of non-amiloride ASIC blockers. DAPI, diminazene, HSB and pentamidine have been tested on $\mathrm{ENaC}$ activities respectively and we found these four compounds (up to $100 \mu \mathrm{M}$ ) were ineffective on $\mathrm{ENaC}$, indicating that DAPI, diminazene, HSB and pentamidine are selective blockers for ASIC over $\mathrm{ENaC}$. These results are in line with the very weak inhibition ( $\mathrm{IC}_{50}: \sim 1 \mathrm{mM}$ ) on $\mathrm{ENaC}$ by nafamostat (Ugawa et al., 2007). Diarylamidines thereby provide alternative pharmacological tools for ASIC study. 
The rank order of apparent inhibitory potencies among the diamidine compounds we tested was diminazene > HSB > DAPI > pentamidine. Pentamidine is connected by a linker that confers structural flexibility (see Table 1) and this may explain its relatively low affinity for inhibition of ASICs. The other three compounds appear to have more rigid structures than pentamidine. Each of these four compounds inhibited ASIC currents, indicating that the linker in each of these diamidines can be variable, while the amidine group linked with an aromatic moiety appears to be essential. Supporting evidence comes from the negative result using netropsin. This compound is also a di-cation that binds to the minor groove of DNA but the charged ends do not directly mount on the aromatic moiety (Table 1) (Nelson et al., 2007). The observation that netropsin failed to inhibit ASICs also suggests that not all DNAbinding divalent cationic agents will inhibit ASICs and even DNA-binding agents need additional structural components in order to bind to ASICs. Furthermore, the weak inhibition by imidocarb suggests that the presence of two charged freely moving termini contribute to the efficient of binding to ASICs.

Tissue acidosis is known to contribute to nociception (Steen et al., 1995) and accumulating evidence has suggested that ASICs participate in acid-induced pain under pathological conditions like inflammation and ischemia (Deval et al., 2008; Xu and Duan, 2009). Thus, ASICs expressed in sensory neurons provide new targets for designing blockers that relieve pain. Although efforts have been made to identify potent ASICs blockers, these efforts have met with limited success (Dube et al., 2005; Ugawa et al., 2007; Kuduk et al., 2009b). We have identified diarylamidines as potent ASICs blockers. Further structure-function studies of these compounds may identify even more potent or subtype-specific inhibitors of ASICs. For example, diminazene display higher inhibitory potency against the currents mediated by ASIC1b, a sensory neuron-specific ASIC subunit (Chen et al., 1998) and ASIC3, a subunit associated primarily with pain sensation (Sluka et al., 2003). Therefore, diminazene may have potential to be used as effective agents for pain suppression. Furthermore, accumulating evidence documents that ASIC channels are emerging as potential therapeutic targets for the alleviation of ischemic stroke (Xiong et al., 2008,, 2004), multiple sclerosis (Friese et al., 2007), anxiety or depression (Sluka et al., 2009; Wemmie et al., 2006). Our study indicates that diarylamidines are leading compounds for future chemical design and screening of potent ASIC inhibitors, and that diarylamidines or similar agentsmay be developed as therapeutic agents to cure ASIC-involved diseases.

\section{Acknowledgments}

We thank L. Brandes and E. Czerwinska for assistance with the primary culture of hippocampal neurons. We appreciate Dr. Harold Atwood for valuable critiques and Dr. Stefan Gründer for helpful comments and generous support for the $\mathrm{ENaC}$ experiments. This work was supported by grants from the Canadian Institutes of Health Research and the Canadian Stroke Network (to J.F.M.), from National Institute of Health (to Z.G.X), and by a postdoctoral research fellowship from the Heart and Stroke Foundation of Canada and the Canadian Stroke Network (to X.C.).

\section{References}

Babini E, Geisler HS, Siba M, Grunder S. A new subunit of the epithelial $\mathrm{Na}^{+}$channel identifies regions involved in $\mathrm{Na}^{+}$self-inhibition. J. Biol. Chem. 2003; 278:28418-28426. [PubMed: 12764146]

Baraldi PG, Bovero A, Fruttarolo F, Preti D, Tabrizi MA, Pavani MG, Romagnoli R. DNA minor groove binders as potential antitumor and antimicrobial agents. Med. Res. Rev. 2004; 24:475-528. [PubMed: 15170593]

Baron A, Waldmann R, Lazdunski M. ASIC-like, proton-activated currents in rat hippocampal neurons. J. Physiol. 2002; 539:485-494. [PubMed: 11882680] 
Chen CC, England S, Akopian AN, Wood JN. A sensory neuron-specific, proton-gated ion channel. Proc. Natl. Acad. Sci. U.S.A. 1998; 95:10240-10245. [PubMed: 9707631]

Chen X, Kalbacher H, Grunder S. The tarantula toxin psalmotoxin 1 inhibits acid-sensing ion channel (ASIC) 1a by increasing its apparent $\mathrm{H}^{+}$affinity. J. Gen. Physiol. 2005; 126:71-79. [PubMed: 15955877]

Chu XP, Wemmie JA, Wang WZ, Zhu XM, Saugstad JA, Price MP, Simon RP, Xiong ZG. Subunitdependent high-affinity zinc inhibition of acid-sensing ion channels. J. Neurosci. 2004; 24:86788689. [PubMed: 15470133]

Deval E, Noel J, Lay N, Alloui A, Diochot S, Friend V, Jodar M, Lazdunski M, Lingueglia E. ASIC3, a sensor of acidic and primary inflammatory pain. EMBO. J. 2008; 27:3047-3055. [PubMed: 18923424]

Diochot S, Baron A, Rash LD, Deval E, Escoubas P, Scarzello S, Salinas M, Lazdunski M. A new sea anemone peptide, APETx2, inhibits ASIC3, a major acid-sensitive channel in sensory neurons. EMBO. J. 2004; 23:1516-1525. [PubMed: 15044953]

Dube GR, Lehto SG, Breese NM, Baker SJ, Wang X, Matulenko MA, Honore P, Stewart AO, Moreland RB, Brioni JD. Electrophysiological and in vivo characterization of A-317567, a novel blocker of acid sensing ion channels. Pain. 2005; 117:88-96. [PubMed: 16061325]

Escoubas P, De Weille JR, Lecoq A, Diochot S, Waldmann R, Champigny G, Moinier D, Menez A, Lazdunski M. Isolation of a tarantula toxin specific for a class of proton-gated $\mathrm{Na}^{+}$channels. J. Biol. Chem. 2000; 275:25116-25121. [PubMed: 10829030]

Friese MA, Craner MJ, Etzensperger R, Vergo S, Wemmie JA, Welsh MJ, Vincent A, Fugger L. Acidsensing ion channel-1 contributes to axonal degeneration in autoimmune inflammation of the central nervous system. Nat. Med. 2007; 13:1483-1489. [PubMed: 17994101]

Gonzales EB, Kawate T, Gouaux E. Pore architecture and ion sites in acid-sensing ion channels and P2X receptors. Nature. 2009; 460:599-604. [PubMed: 19641589]

Grunder S, Geissler HS, Bassler EL, Ruppersberg JP. A new member of acid-sensing ion channels from pituitary gland. Neuroreport. 2000; 11:1607-1611. [PubMed: 10852210]

Hermans PE, Keys TF. Antifungal agents used for deep-seated mycotic infections. Mayo Clin. Proc. 1983; 58:223-231. [PubMed: 6339832]

Jasti J, Furukawa H, Gonzales EB, Gouaux E. Structure of acid-sensing ion channel 1 at $1.9 \AA$ resolution and low pH. Nature. 2007; 449:316-323. [PubMed: 17882215]

Kellenberger S, Schild L. Epithelial sodium channel/degenerin family of ion channels: a variety of functions for a shared structure. Physiol. Rev. 2002; 82:735-767. [PubMed: 12087134]

Kinabo LD. Pharmacology of existing drugs for animal trypanosomiasis. Acta Trop. 1993; 54:169183. [PubMed: 7902656]

Krishtal O. The ASICs: signaling molecules? Modulators? Trends Neurosci. 2003; 26:477-483. [PubMed: 12948658]

Kuduk SD, Chang RK, Wai JM, Di Marco CN, Cofre V, DiPardo RM, Cook SP, Cato MJ, Jovanovska A, Urban MO, Leitl M, Spencer RH, Kane SA, Hartman GD, Bilodeau MT. Amidine derived inhibitors of acid-sensing ion channel-3 (ASIC3). Bioorg. Med. Chem. Lett. 2009a; 19:40594063. [PubMed: 19553111]

Kuduk SD, Di Marco CN, Chang RK, Dipardo RM, Cook SP, Cato MJ, Jovanovska A, Urban MO, Leitl M, Spencer RH, Kane SA, Bilodeau MT, Hartman GD, Bock MG. Amiloride derived inhibitors of acid-sensing ion channel-3 (ASIC3). Bioorg. Med. Chem. Lett. 2009b; 19:25142518. [PubMed: 19339181]

Lingueglia E. Acid-sensing ion channels in sensory perception. J. Biol. Chem. 2007; 282:1732517329. [PubMed: 17430882]

Mishra J, Saxena A, Singh S. Chemotherapy of leishmaniasis: past, present and future. Curr. Med. Chem. 2007; 14:1153-1169. [PubMed: 17456028]

Nelson SM, Ferguson LR, Denny WA. Non-covalent ligand/DNA interactions: minor groove binding agents. Mutat. Res. 2007; 623:24-40. [PubMed: 17507044]

Paukert M, Babini E, Pusch M, Grunder S. Identification of the $\mathrm{Ca}^{2+}$ blocking site of acid-sensing ion channel (ASIC) 1: implications for channel gating. J. Gen. Physiol. 2004; 124:383-394. [PubMed: 15452199] 
Paukert M, Chen X, Polleichtner G, Schindelin H, Grunder S. Candidate amino acids involved in $\mathrm{H}^{+}$ gating of acid-sensing ion channel 1a. J. Biol. Chem. 2008; 283:572-581. [PubMed: 17981796]

Rosenthal, P. Antiprotozoal Drugs. Mcgraw-Hill; 2007.

Sluka KA, Price MP, Breese NM, Stucky CL, Wemmie JA, Welsh MJ. Chronic hyperalgesia induced by repeated acid injections in muscle is abolished by the loss of ASIC3, but not ASIC1. Pain. 2003; 106:229-239. [PubMed: 14659506]

Sluka KA, Winter OC, Wemmie JA. Acid-sensing ion channels: a new target for pain and CNS diseases. Curr. Opin. Drug Discov. Dev. 2009; 12:693-704.

Steen KH, Issberner U, Reeh PW. Pain due to experimental acidosis in human skin: evidence for nonadapting nociceptor excitation. Neurosci. Lett. 1995; 199:29-32. [PubMed: 8584219]

Ugawa S, Ishida Y, Ueda T, Inoue K, Nagao M, Shimada S. Nafamostat mesilate reversibly blocks acid-sensing ion channel currents. Biochem. Biophys. Res. Commun. 2007; 363:203-208. [PubMed: 17826743]

Ugawa S, Ueda T, Ishida Y, Nishigaki M, Shibata Y, Shimada S. Amiloride-blockable acid-sensing ion channels are leading acid sensors expressed in human nociceptors. J. Clin. Invest. 2002; 110:1185-1190. [PubMed: 12393854]

Voilley N, de Weille J, Mamet J, Lazdunski M. Nonsteroid anti-inflammatory drugs inhibit both the activity and the inflammation-induced expression of acid-sensing ion channels in nociceptors. J. Neurosci. 2001; 21:8026-8033. [PubMed: 11588175]

Waldmann R, Champigny G, Bassilana F, Heurteaux C, Lazdunski M. A proton-gated cation channel involved in acid-sensing. Nature. 1997; 386:173-177. [PubMed: 9062189]

Wei WL, Sun HS, Olah ME, Sun X, Czerwinska E, Czerwinski W, Mori Y, Orser BA, Xiong ZG, Jackson MF, Tymianski M, MacDonald JF. TRPM7 channels in hippocampal neurons detect levels of extracellular divalent cations. Proc. Natl. Acad. Sci. U.S.A. 2007; 104:16323-16328. [PubMed: 17913893]

Wemmie JA, Price MP, Welsh MJ. Acid-sensing ion channels: advances, questions and therapeutic opportunities. Trends Neurosci. 2006; 29:578-586. [PubMed: 16891000]

Wilson WD, Nguyen B, Tanious FA, Mathis A, Hall JE, Stephens CE, Boykin DW. Dications that target the DNA minor groove: compound design and preparation, DNA interactions, cellular distribution and biological activity. Curr. Med. Chem. Anticancer Agents. 2005; 5:389-408. [PubMed: 16101490]

Wu LJ, Duan B, Mei YD, Gao J, Chen JG, Zhuo M, Xu L, Wu M, Xu TL. Characterization of acidsensing ion channels in dorsal horn neurons of rat spinal cord. J. Biol. Chem. 2004; 279:4371643724. [PubMed: 15302881]

Xiong ZG, Pignataro G, Li M, Chang SY, Simon RP. Acid-sensing ion channels (ASICs) as pharmacological targets for neurodegenerative diseases. Curr. Opin. Pharmacol. 2008; 8:25-32. [PubMed: 17945532]

Xiong ZG, Zhu XM, Chu XP, Minami M, Hey J, Wei WL, MacDonald JF, Wemmie JA, Price MP, Welsh MJ, Simon RP. Neuroprotection in ischemia: blocking calcium-permeable acid-sensing ion channels. Cell. 2004; 118:687-698. [PubMed: 15369669]

$\mathrm{Xu}$ TL, Duan B. Calcium-permeable acid-sensing ion channel in nociceptive plasticity: a new target for pain control. Prog. Neurobiol. 2009; 87:171-180. [PubMed: 19388206]

Ziemann AE, Schnizler MK, Albert GW, Severson MA, Howard MA 3rd, Welsh MJ, Wemmie JA. Seizure termination by acidosis depends on ASIC1a. Nat. Neurosci. 2008; 11:816-822. [PubMed: 18536711] 
A

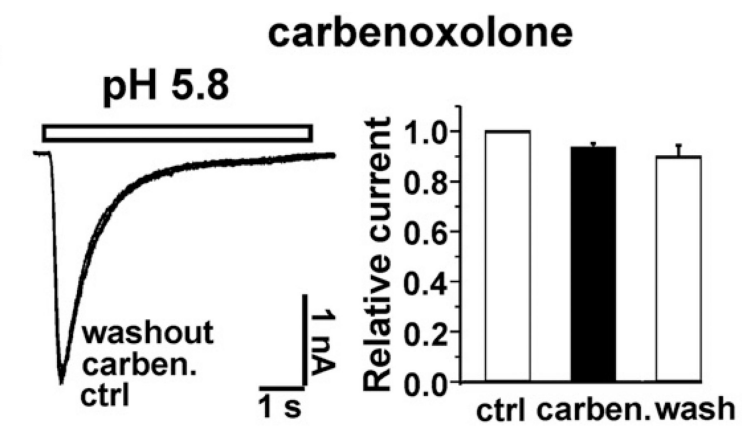

B

netropsin

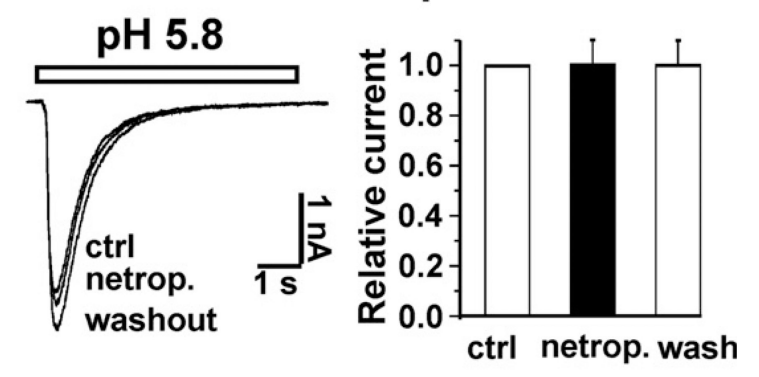

C
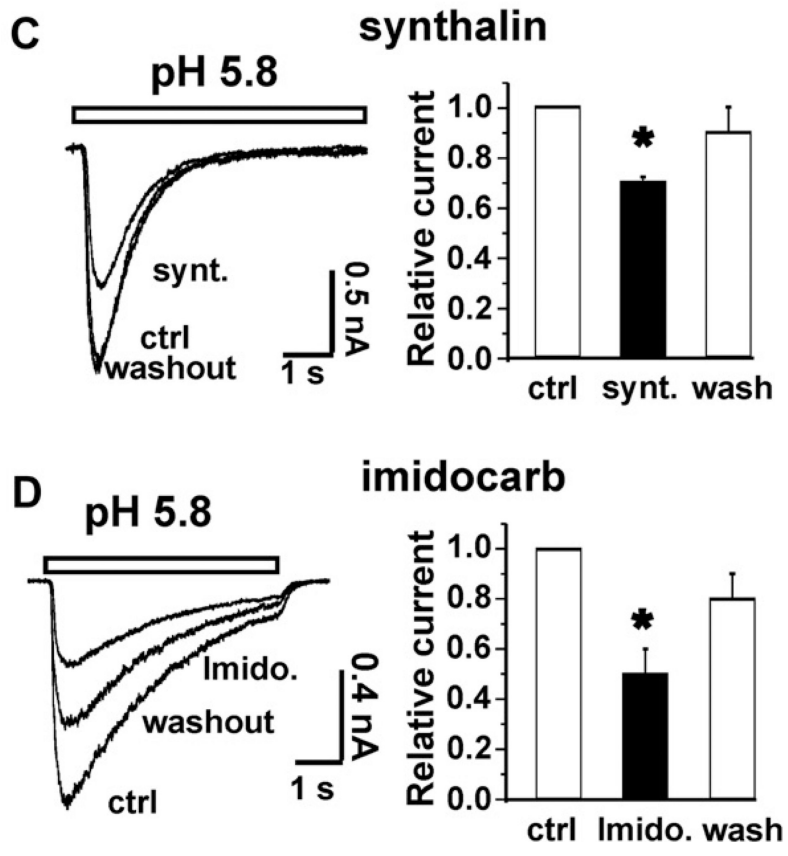

Fig. 1.

Chemicals that have no effect, or only have partial effect, on ASIC activities in cultured hippocampal neurons. A. Carbenoxolone $(200 \mu \mathrm{M})$ did not inhibit ASIC activities. Left, exemplary current trace overlay; right, bar graph of relative current without and with carbenxolone. $n=4, p=0.1$. B. Netropsin $(100 \mu \mathrm{M})$ did not inhibit ASIC activities. Left, exemplary current trace overlay; right, bar graph showing relative current without and with netropsin, $n=6, p=0.24$. C. Synthalin $(200 \mu \mathrm{M})$ inhibited $30 \pm 2 \%(n=6, p<0.01)$ of ASIC activities. Left, exemplary current trace overlay; right, bar graph of relative current without and with synthalin. D. Imidocarb $(200 \mu \mathrm{M})$ inhibited $42 \pm 7 \%(n=5, p<0.05)$ of 
ASIC currents. Left, exemplary current trace overlay; right, bar graph showing relative current without and with imidocarb. 
$\mathbf{A}$
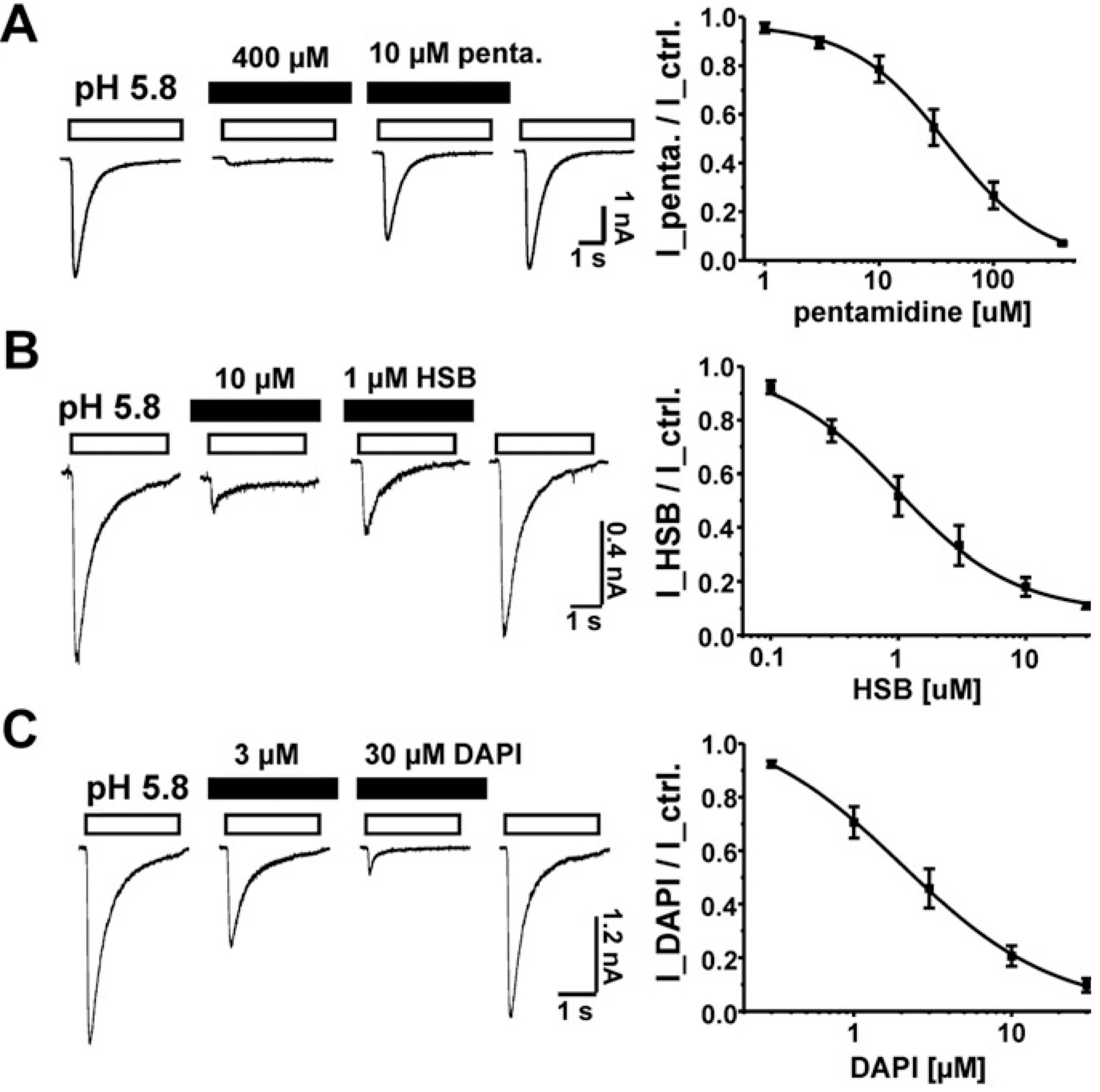

Fig. 2.

Pentamidine, HSB and DAPI reversibly blocked ASIC activities in cultured hippocampal neurons. A. Pentamidine inhibited ASIC current. Left, representative current traces; right, concentration response. $\mathrm{IC}_{50}: 38 \pm 11 \mu \mathrm{M}, n=4$. B. HSB inhibited ASIC activities in cultured hippocampal neurons. Left, exemplary current traces; right, concentration response. $\mathrm{IC}_{50}: 1.5 \pm 0.6 \mu \mathrm{M}, n=5$. C. DAPI inhibited ASIC current. Left, representative current traces; right, concentration response. $\mathrm{IC}_{50}, 2.8 \pm 0.7 \mu \mathrm{M}, n=4$. 


\section{A $\quad 0.1 \mu \mathrm{M} \quad 3 \mu \mathrm{M}$ dimi.}

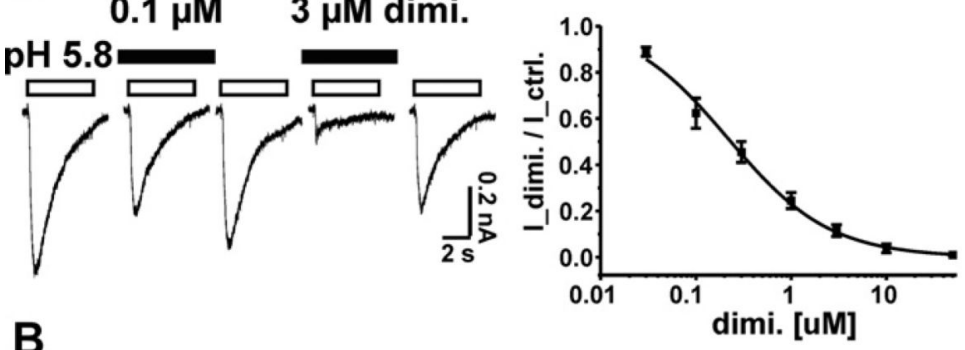

B

pH $5.83 \mu \mathrm{M}$ dimi.

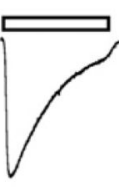

C
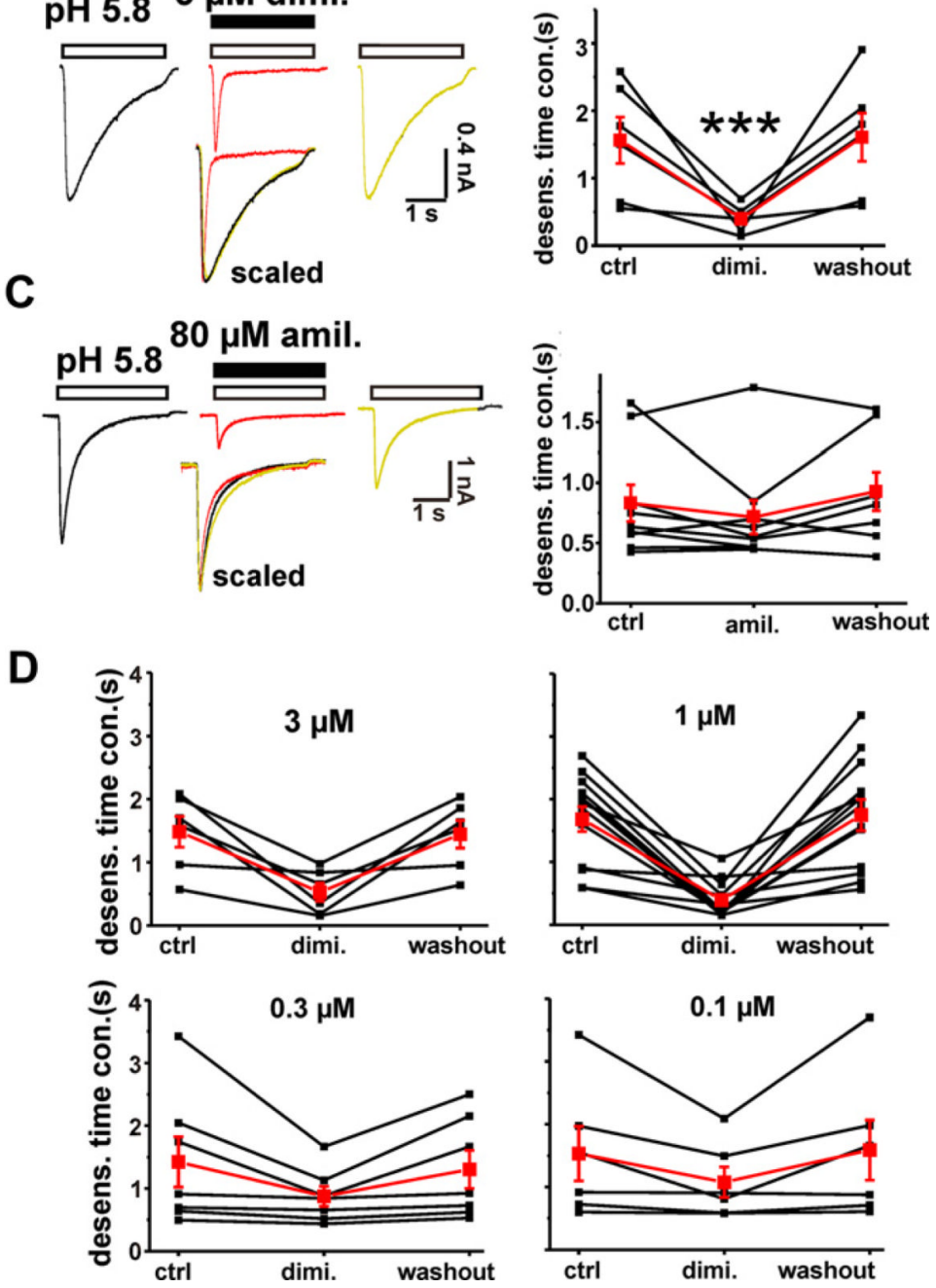

Fig. 3.

Diminazene potently inhibited ASIC current in cultured hippocampal neurons. A. Diminazene blocked ASIC. Left, representative ASIC current traces with and without diminazene. ASIC current was evoked by application of $\mathrm{pH} 5.8$; right, concentration response of ASIC inhibition by diminazene. IC50: $0.28 \pm 0.11 \mu \mathrm{M}, n=6$. B. Co-applied diminazene promoted the closure of ASIC currents. Left, representative current traces. Coapplied diminazene $(3 \mu \mathrm{M})$ with $\mathrm{pH} 5.8$ drastically accelerated the decay of desensitization of AISC currents; right, statistics of desensitization time constant; control $1.6 \pm 0.3 \mathrm{~s}$; with diminazene, $0.4 \pm 0.1 \mathrm{~s}$ and washout $1.6 \pm 0.4 \mathrm{~s}, n=6, p<0.001$. C. Sub-maximal 
concentration of amiloride $(80 \mu \mathrm{M}$, co-applied with $\mathrm{pH}$ 5.8) did not accelerate the desensitization of ASIC current. Left, current trace examples; right, statistics of desensitization time constant with and without amiloride. D. Sub-maximal concentrations of diminazene (by perfusion) significantly accelerated the desensitization of ASIC current.

Statistics of desensitization time constant of ASIC at the absence or presence of $3 \mu \mathrm{M}, 1 \mu \mathrm{M}$, $0.3 \mu \mathrm{M}$ and $0.1 \mu \mathrm{M}$ diminazene, respectively. 
A
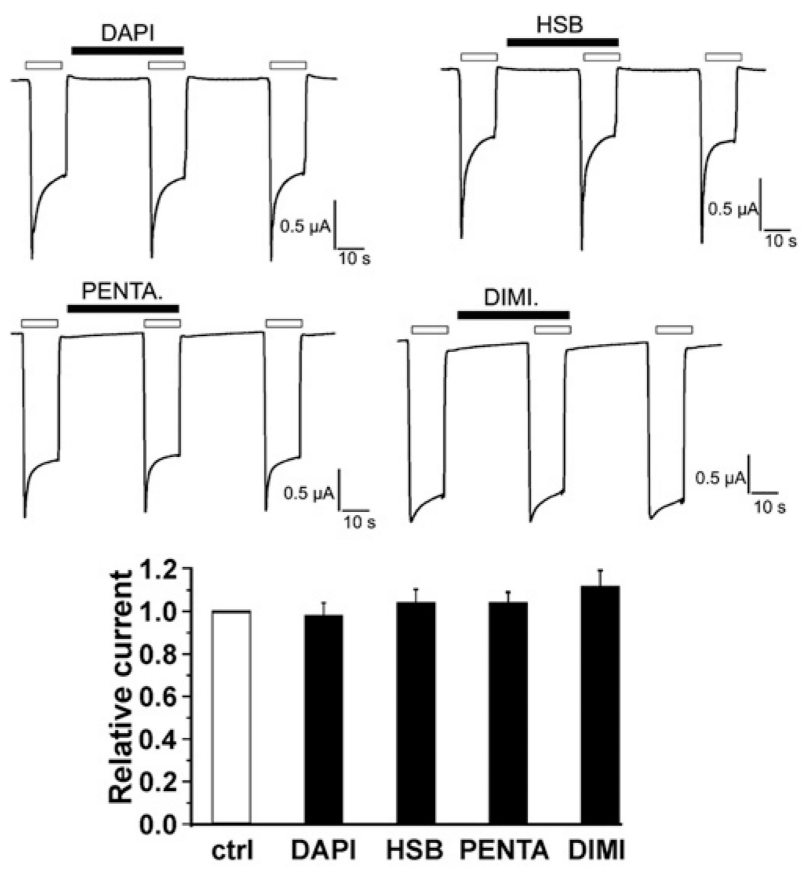

B
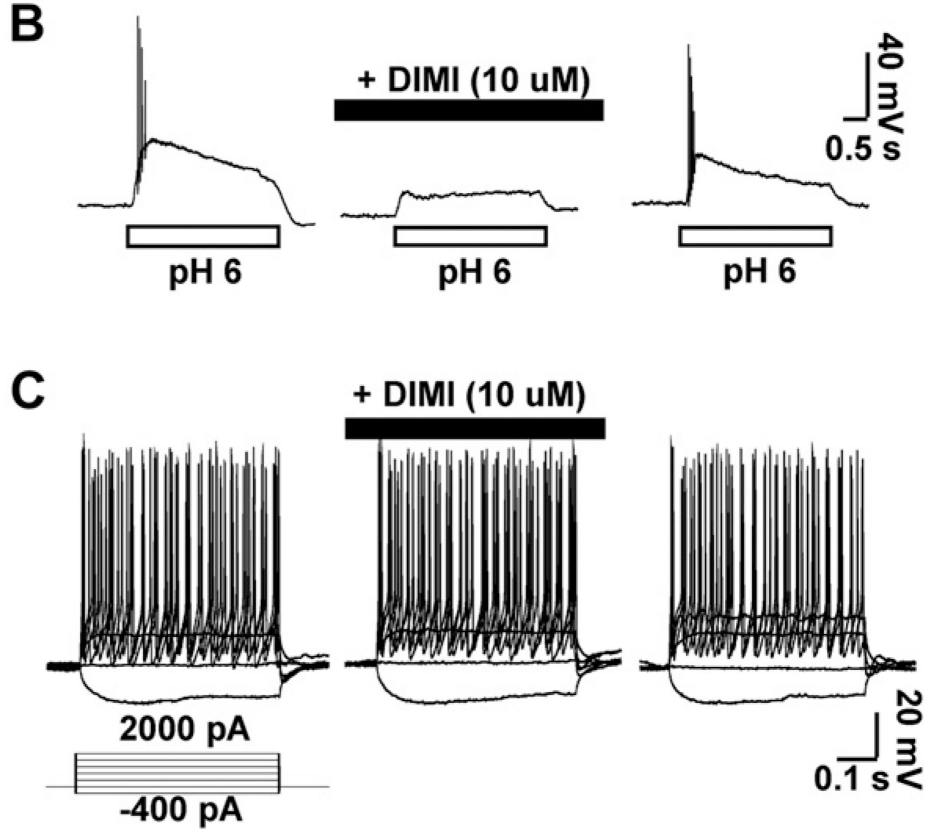

Fig. 4.

Diarylamidines failed to block oocyte-expressing ENaC channels. A. DAPI, HSB, pentamidine and diminazene (at $100 \mu \mathrm{M}$, respectively) did not inhibit ENaC activities. $\mathrm{ENaC}$ channels were blocked by perfusion of $10 \mu \mathrm{M}$ amiloride, brief washout of amiloride (indicated by white bars) revealed $\mathrm{ENaC}$ activities. Top, after control, diarylamidines (black bars) were applied before and during amiloride-washout; Bottom, bar graph of relative currents of $\mathrm{ENaC}$ with and without diarylamidines. $\mathrm{ENaC}$ activities were not significantly affected by DPAI $(0.98 \pm 0.04, n=8, p=0.69)$, by HSB $(1.02 \pm 0.04, n=8, p=0.55)$, by pentamidine $(1.02 \pm 0.03, n=8, p=0.57)$ or by diminazene $(1.11 \pm 0.05, n=8, p=0.06)$. 
B. Diminazene attenuated low pH-induced neuronal excitability. Application of pH 5.8 to neuron caused action potentials and sustained depolarization; addition of diminazene (10 $\mu \mathrm{M})$ markedly attenuated membrane depolarization. $n=7$. C. Current-clamp recording. Action potentials were elicited by current injection. Application of diminazene $(10 \mu \mathrm{M}) \mathrm{did}$ not affect action potentials. $n=4$. 

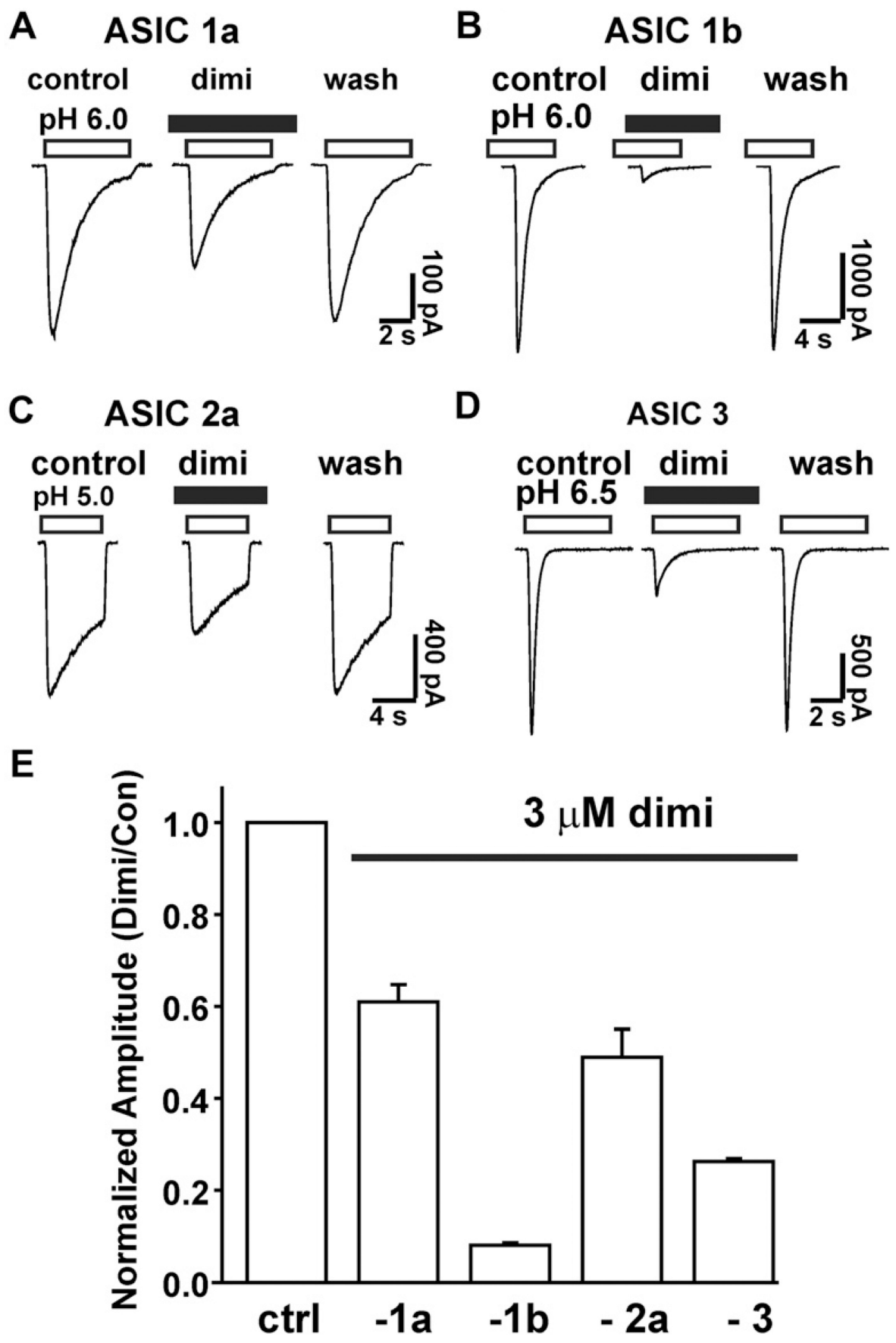

Fig. 5.

Diminazene inhibited ASIC currents mediated by individual ASIC subunit expressed in CHO cells. Diminazene $(3 \mu \mathrm{M})$ reversibly inhibited the ASIC currents mediated by homomeric ASIC1a (A), homomeric ASIC1b (B), homomeric ASIC2a (C), and homomeric ASIC3 channels (D) expressed in CHO cells. E. Summary bar graph showing the inhibition of the ASIC1a, 1b, 2a, or 3 current by $3 \mu \mathrm{M}$ diminazene. ASIC1a current was reduced by 39 $\pm 3.8 \%(n=7, p<0.01)$, ASIC1b current by $92 \pm 0.5 \%(n=8, p<0.01)$, ASIC2a current by $51 \pm 6.1 \%(n=8, p<0.01)$, and ASIC3 current by $74 \pm 0.6 \%(n=7, p<0.01)$, respectively. 


\section{A diminazene}

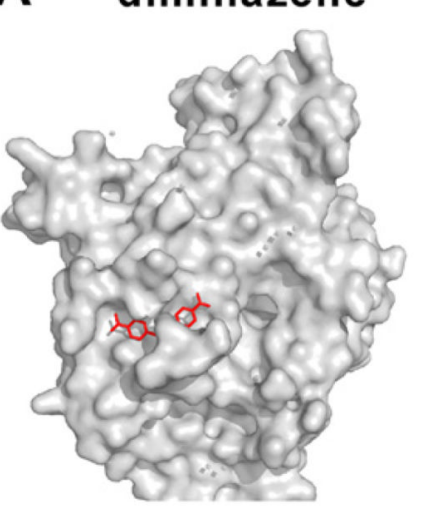

DAPI

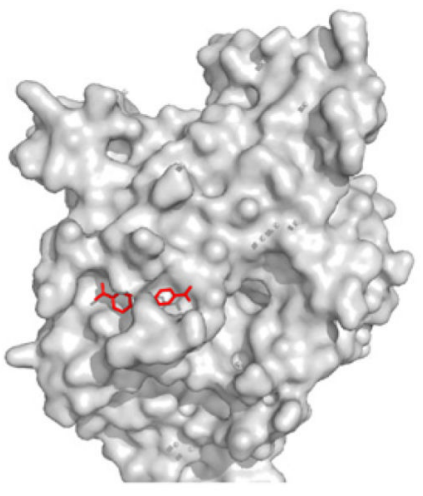

\section{B diminazene}

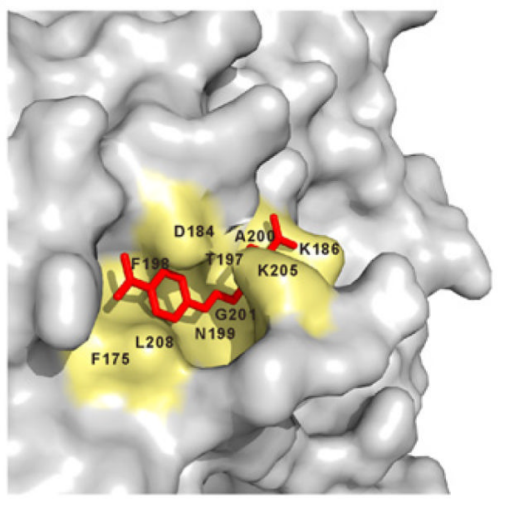

Fig. 6.
HSB

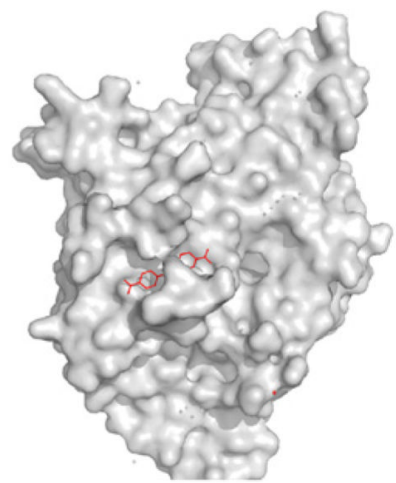

pentamidine

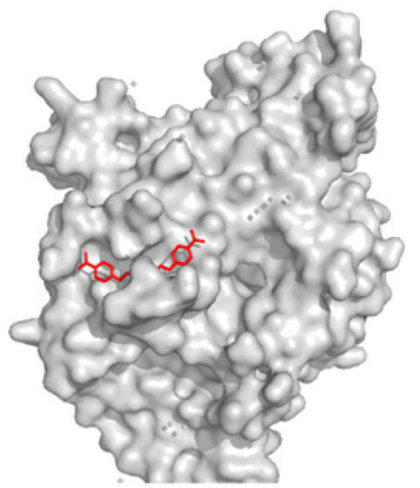

C diminazene

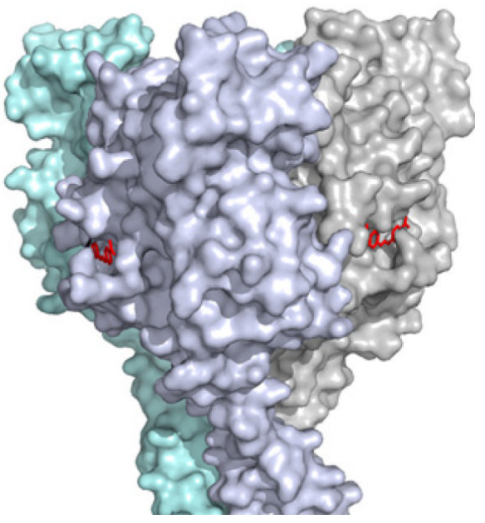

Docking diarylamidines onto cASIC1 crystal structure. A. Diminazene, HSB, DAPI and pentamidine commonly docked to a groove-shaped domain onto ASIC1. Crystal structure of ASIC1 and ASIC blockerswere uploaded into the server, respectively and dock computation was performed by the pacthdock server (http://bioinfo3d.cs.tau.ac.il/PatchDock/). B.

Enlargement of the binding domain with diminazene bound. Individual amino acids in the binding domain as well as their positions in protein sequence were shown. The bottom part of the groove was formed by residues F175, K186, T197, F198, N199, A200, G201, and L208. The hydrophobic bottom part of the groove is large so that it accommodates sizevariation of these blockers. Charged amino acids D184 and K205 control the upper 
restrictive entrance of the binding region. C. Steric side-view of ASIC channel. Diminazene docked onto the same place in the trimeric channel. Three subunits of ASIC channel are shown in distinct colors. (For interpretation of the references to color in this figure legend, the reader is referred to the web version of this article.) 
Table 1

Comparing known ASIC inhibitors with diarylamidines.

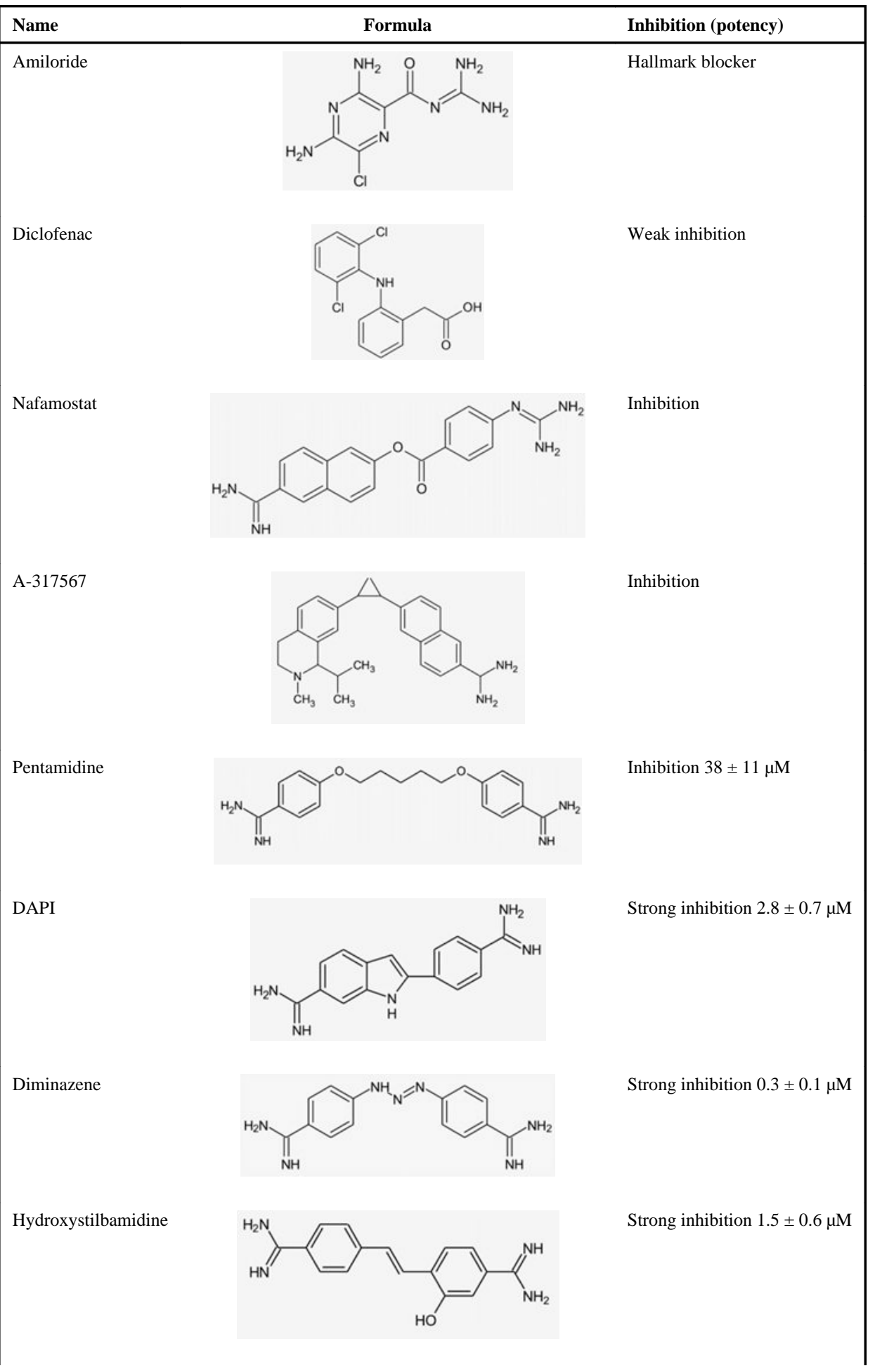




Name Imidocarb

